\title{
Relationship Between Tau and Cognition in the Evolution of Alzheimer's Disease: New Insights from Tau PET
}

\author{
Jeremy A. Tanner ${ }^{1}$ and Gil D. Rabinovici ${ }^{1,2}$ \\ ${ }^{I}$ Memory and Aging Center, Department of Neurology, University of California San Francisco, San Francisco, California; and \\ ${ }^{2}$ Department of Radiology and Biomedical Imaging, University of California San Francisco, San Francisco, California
}

$\mathbf{W}$

ith the recent approval of ${ }^{18} \mathrm{~F}$-flortaucipir (previously called T807/AV-1451, trade name Tauvid; Lilly) for clinical use by the U.S. Food and Drug Administration, there is increasing excitement around tau imaging. ${ }^{18} \mathrm{~F}$-flortaucipir PET is now approved to estimate the density and distribution of aggregated tau neurofibrillary tangles in adults with cognitive impairment undergoing evaluation for Alzheimer's disease (AD) (1). The Food and Drug Administration approval was based on evidence that visual interpretations of ${ }^{18} \mathrm{~F}$-flortaucipir PET showed high sensitivity and specificity for advanced-stage tau pathology as determined at autopsy (2). In addition to its diagnostic use, tau PET can image the spatiotemporal progression of tau pathology in longitudinal studies, shining a light on the central role of tau in driving neurodegeneration and cognitive decline throughout the insidious evolution of $\mathrm{AD}$.

Tau is a microtubule binding protein that plays an important physiologic role in axonal transport, cytoskeletal architecture, and membrane-based signaling pathways (3). In humans, tau undergoes alternative splicing to form 6 isoforms, each of which contains either 3 or 4 repeat microtubule binding motifs (3R or $4 \mathrm{R}$ isoforms). In neurodegenerative diseases, tau undergoes abnormal phosphorylation and acetylation, causing it to disassemble from microtubules and form fibrillar insoluble aggregates that propagate through the brain. In $\mathrm{AD}$, aggregated forms of tau are found in dystrophic neurites around amyloid- $\beta$ plaques and in neurofibrillary tangles that consist of mixed $3 \mathrm{R} / 4 \mathrm{R}$ tau isoforms that form paired helical filaments. ${ }^{18} \mathrm{~F}$-flortaucipir, the first and most widely studied tau-specific radiotracer, is specific to the tau aggregates of $\mathrm{AD}$ and shows absent-to-low binding in non- $\mathrm{AD}$ tauopathies. Autopsy studies have shown that neurofibrillary tangles follow a stereotypical progression from transentorhinal (Braak stages I/II) to limbic (Braak III/IV) and finally neocortical (Braak V/VI) regions. Tau PET patterns reported with ${ }^{18} \mathrm{~F}$-flortaucipir and other tau radiotracers generally reproduce the expected topography of neuropathologic Braak staging (4).

$\mathrm{AD}$ neuropathologic changes are measured based on the distribution of both amyloid and tau pathology. The relationship between $\mathrm{AD}$ neuropathologic changes and clinical state is

Received Nov. 15, 2020; revision accepted Dec. 1, 2020.

For correspondence or reprints contact: Jeremy A. Tanner, UCSF Memory and Aging Center, Box 1207, 675 Nelson Rising Lane, Suite 190, San Francisco, CA 94143.

E-mail: jeremy.tanner@ucsf.edu

Published online Dec. 4, 2020.

COPYRIGHT (c) 2021 by the Society of Nuclear Medicine and Molecular Imaging. DOI: 10.2967/jnumed.120.257824 complex. It is now widely recognized that $\mathrm{AD}$ has a prolonged preclinical phase, characterized by the presence of plaques and tangles without clinically significant cognitive changes. Over time, many individuals with preclinical AD develop mild cognitive impairment (defined by objectively confirmed cognitive deficits) and ultimately dementia (defined by functional impairment). Clinicopathologic studies have revealed that cognition and clinical state are much more closely related to the distribution and burden of neurofibrillary tangles than to the topography or degree of amyloid plaques (5). The relationship between AD neuropathologic changes and cognition is further modified by patient-specific factors including age, sex, education, genetics, and additional brain copathologies.

As would be expected from neuropathology, worsening disease stage in $\mathrm{AD}$ is associated with increased intensity and spread of tau PET ligand retention. In cognitively normal older adults, increased age is associated with increased tau PET binding in the medial temporal lobes independent of amyloid status, as observed in autopsy studies in Braak I/II regions (4). In amyloid-positive preclinical AD (defined on the basis of PET or other biomarker evidence of both amyloid and tau), tau PET retention increases in the medial temporal lobe and spreads further into the inferiorlateral temporal cortex (4). With progression to mild cognitive impairment, tau signal extends farther into the lateral temporal and posterior cingulate cortices (6). Progression to the dementia stage is characterized by increased intensity and spread of binding to the parietal and dorsolateral prefrontal cortices, with relative preservation of primary unimodal cortices (6).

Throughout the course of disease, both the distribution and the burden of tau PET binding correlate with cognitive symptoms. In preclinical $\mathrm{AD}$ and mild cognitive impairment, increased medial and inferior temporal tau is associated with decreased performance on episodic memory tests (7-9). Figure 1 illustrates that in patients with $\mathrm{AD}$, the topography of ${ }^{18} \mathrm{~F}$-flortaucipir binding correlates with performance in specific cognitive domains according to established brain-behavior relationships (i.e., language in the left perisylvian region, episodic memory in the medial temporal lobe, semantic memory in the left anterior temporal lobe, executive function in the right and left frontoparietal cortex, and visuospatial in the occipital and posterior parietal cortex) (10). Relatedly, patients with logopenic-variant primary progressive aphasia (a language-predominant variant of $\mathrm{AD}$ ) show asymmetric left-hemisphere tau PET binding, whereas patients with posterior cortical atrophy (a visuospatial-predominant variant) show increased occipital binding (11). Notably, amyloid PET patterns show absent-to-weak correlations with cognitive deficits and the clinical AD phenotype. 


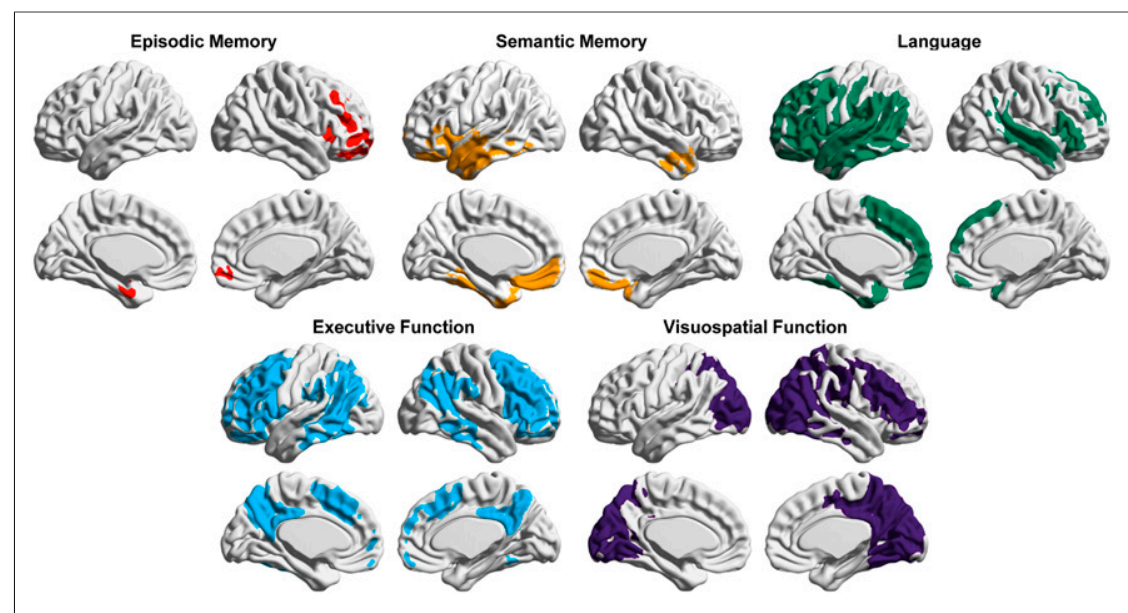

FIGURE 1. Voxelwise analysis showing correlation between increased ${ }^{18} \mathrm{~F}$-flortaucipir SUVR and decreased domain-specific cognitive performance in clinically heterogeneous sample of 93 patients with $\mathrm{AD}$. Voxels are significant at peak-level threshold of $P<0.001$ uncorrected, clusterlevel Family-wise error corrected $P<0.05$.

Multiple factors affect the relationship between tau, brain structure, and cognition in AD. Younger age at disease onset is associated with increased tau burden but also greater cognitive and brain resilience, where early-onset $\mathrm{AD}$ patients (age $\leq 65 \mathrm{y}$ ) have a greater tau burden relative to their disease stage than patients with late-onset $\mathrm{AD}(9,11,12)$. Women have a greater tau burden but also greater preservation of cognition and brain structure relative to tau than men (12). Patients who carry the apolipoprotein E $\varepsilon 4$ risk allele have a greater tau accumulation in the medial temporal lobe than patients with other apolipoprotein E genotypes $(9,11)$. A higher educational level is associated with greater cognitive resilience relative to tau burden (12).

Multimodal imaging analyses have provided insights into the relationship between amyloid, tau, neurodegeneration, and cognition. Growing evidence suggests that amyloid PET positivity is critical for tau spread from the medial temporal lobe to the neocortex $(4,9)$. Tau imaging is strongly correlated with neurodegeneration (i.e., gray matter atrophy on MRI or hypometabolism on ${ }^{18}$ F-FDG PET), which partially mediates the localized effect of tau PET on cognition (10). Vascular dysfunction also affects the relationship between tau and cognition and provides an additional target for clinical intervention (13). Studies combining tau PET with MRI structural or functional connectivity measures support the notion that tau aggregates propagate through transneuronal connections (14), supporting therapeutic interventions such as tau-targeting monoclonal antibodies designed to slow or prevent tau spread.

Recently, longitudinal studies have revealed that tau PET predicts both future neurodegeneration and clinical decline. Baseline tau PET predicts the rate and topography of future gray matter atrophy at the single-patient level, independent of baseline atrophy and amyloid (15). Tau PET is associated with future cognitive decline across the AD clinical spectrum (9), and spread aligns with conversion from preclinical AD to mild cognitive impairment (8) and from mild cognitive impairment to dementia (16). As a biomarker of both neuropathology and disease progression, tau PET has many roles in AD clinical trials including subject selection, group stratification (e.g., higher baseline tau PET predictive of more rapid progression), measurement of target engagement (for tau-directed therapies), and measurement of downstream disease modification (e.g., for amyloid-directed therapies).

In summary, tau imaging enables in vivo visualization of $\mathrm{AD}$ molecular pathology that localizes with cognitive symptoms and aligns with clinical severity. Therefore, tau PET is likely to enhance precision medicine approaches to AD diagnosis, prognostication, drug development, and future clinical care. Ultimately, the close association between tau imaging and cognition has strengthened hope that future antitau therapeutics will slow cognitive decline in AD.

\section{DISCLOSURE}

Gil Rabinovici receives research support from Avid Radiopharmaceuticals, Eli Lilly, GE Healthcare, and Life Molecular Imaging. He has received consultation fees from Axon Neurosciences, Eisai, and Merck. He serves on a data safety monitoring board for Johnson \& Johnson and as an associate editor for JAMA Neurology. No other potential conflict of interest relevant to this article was reported.

\section{REFERENCES}

1. Barthel H. First tau PET tracer approved: toward accurate in vivo diagnosis of Alzheimer disease. J Nucl Med. 2020;61:1409-1410.

2. Fleisher AS, Pontecorvo MJ, Devous MD, et al. Positron emission tomography imaging with $\left[{ }^{18} \mathrm{~F}\right]$ flortaucipir and postmortem assessment of Alzheimer disease neuropathologic changes. JAMA Neurol. 2020;77:829-839.

3. Morris M, Maeda S, Vossel K, Mucke L. The many faces of tau. Neuron. 2011;70:410-426.

4. Schöll M, Lockhart SN, Schonhaut DR, et al. PET imaging of tau deposition in the aging human brain. Neuron. 2016;89:971-982.

5. Nelson PT, Alafuzoff I, Bigio EH, et al. Correlation of Alzheimer disease neuropathologic changes with cognitive status: a review of the literature. J Neuropathol Exp Neurol. 2012;71:362-381.

6. Pontecorvo MJ, Devous MD, Navitsky M, et al. Relationships between flortaucipir PET tau binding and amyloid burden, clinical diagnosis, age and cognition. Brain. 2017;140:748-763.

7. Maass A, Lockhart SN, Harrison TM, et al. Entorhinal tau pathology, episodic memory decline, and neurodegeneration in aging. J Neurosci. 2018;38:530-543.

8. Hanseeuw BJ, Betensky RA, Jacobs HIL, et al. Association of amyloid and tau with cognition in preclinical Alzheimer disease. JAMA Neurol. 2019;76:915-924.

9. Jack CR, Wiste HJ, Weigand SD, et al. Predicting future rates of tau accumulation on PET. Brain. 2020;143:3136-3150.

10. Bejanin A, Schonhaut DR, La Joie R, et al. Tau pathology and neurodegeneration contribute to cognitive impairment in Alzheimer's disease. Brain. 2017;140:3286-3300.

11. Ossenkoppele R, Schonhaut DR, Scholl M, et al. Tau PET patterns mirror clinical and neuroanatomical variability in Alzheimer's disease. Brain. 2016;139:1551-1567.

12. Ossenkoppele R, Lyoo CH, Jester-Broms J, et al. Assessment of demographic, genetic, and imaging variables associated with brain resilience and cognitive resilience to pathological tau in patients with Alzheimer disease. JAMA Neurol. 2020;77:632-642.

13. Albrecht D, Isenberg AL, Stradford J, et al. Associations between vascular function and tau PET are associated with global cognition and amyloid. J Neurosci. 2020;40:8573-8586.

14. Vogel JW, Iturria-Medina Y, Strandberg OT, et al. Spread of pathological tau proteins through communicating neurons in human Alzheimer's disease. Nat Commun. 2020;11:2612.

15. La Joie R, Visani AV, Baker SL, et al. Prospective longitudinal atrophy in Alzheimer's disease correlates with the intensity and topography of baseline tau-PET. Sci Transl Med. 2020;12:eaau5732.

16. Cho H, Choi JY, Lee HS, et al. Progressive tau accumulation in Alzheimer disease: 2-year follow-up study. J Nucl Med. 2019;60:1611-1621. 